\title{
O uso de funções na geração de novas alturas sonoras, a partir de um conjunto de classes de nota
}

\author{
The use of functions in the generation of new pitches, from a pitch- \\ class set
}

\author{
Pedro Henrique Carneiro Tavares \\ Conservatório Pernambucano de Música. E-mail: phtmetal@gmail.com
}

\section{RESUMO}

0 artigo apresenta resultados de pesquisa acerca da aplicação de funções na geração de novas alturas sonoras, a partir de um conjunto de classes de nota original. São apresentados conceitos tanto do âmbito matemático quanto musical, no tocante a matemática são citados funções e teoria dos conjuntos, enquanto no âmbito musical são apresentados conceitos utilizados na teoria póstonal, particularmente classes de nota e conjuntos de classes de nota. Além da aplicação das funções na geração de novas alturas sonoras, são apresentadas, com finalidade de demonstração e sugestão de aplicação prática, frases musicais desenvolvidas a partir dos resultados da pesquisa.

Palavras-Chave: Funções, Teoria dos conjuntos, Classes de nota.

\section{ABSTRACT}

The article presents research results about the application of functions in the generation of new pitches, from a pitch-class set. Concepts from both the mathematical and musical fields are presented, with respect to mathematics, functions and set theory are cited, while in the musical field, concepts used in post-tonal theory are presented, particularly pitch-class and pitch-class set theory. In addition to the application of functions in the generation of new pitches, musical phrases developed from the research results are presented, with purpose of demonstration and suggestion of practical application.

Keywords: Functions, Set theory, Pitch-class. 
TAVARES, Pedro Henrique Carneiro (2021)

$O$ uso de funções na geração de novas alturas sonoras, a partir de um conjunto de classes de nota.

\section{Introdução}

A partir do início do século $X X$, e seguindo para o século $X X I$, um grande número de novas estéticas e procedimentos foram desenvolvidos no campo musical. Parte desses procedimentos se enquadram no campo de estudo da chamada teoria pós-tonal, que, por exemplo, estuda elementos como o uso de conjuntos de classe de nota, a série dodecafônica, procedimentos seriais, dentre outros.

Através do estudo da teoria pós-tonal, particularmente acerca da aplicação da teoria dos conjuntos de classes de nota no processo de criação musical, houve a constatação de que, entre os procedimentos empregados com conjuntos, não foi possível encontrar nenhum exemplo do uso de funções. Dentre os procedimentos mais constantes revelam-se a transposição, inversão, multiplicação, permutação, etc. Os três primeiros procedimentos (Transposição, inversão e multiplicação) são utilizados, por exemplo, no trabalho publicado por Pedro e Maria Clara Tavares (2021), sobre o uso combinado de Modelagem Sistêmica e Teoria dos Conjuntos no âmbito de Composição Musical. Em quantidade menor de casos se pode observar outras abordagens, tais como, por exemplo, a permutação serial cíclica de Pousseur, explanada em detalhes por Menezes (2002. p.304-316).

Partindo da curiosidade despertada pela constatação citada, o presente trabalho foi desenvolvido no intuito de apresenta reflexões e sugestões práticas acerca da temática: geração de novas classes de nota através do uso de funções, a partir de um conjunto de classes de nota. Inicialmente estabelecemos os conceitos de conjunto, função, classe de nota e conjunto de classes de nota.

A Teoria dos Conjuntos remete à discussões difíceis no âmbito conceitual da matemática, tal qual a definição de número. Publicações como as de Richard Dudekin, particularmente Stetigkeit und Irrationale Zahlen (Continuidade e números irracionais), de 1872, e Was Sind und was Sollen die Zahlen? (0 que são e o que significam os números?), de 1888, trazem destaque à temática. Dentre outros trabalhos, é importante destacar a contribuição de Gottlob Frege, que, de maneira resumida, propõe uma associação de números com contagem, tal abordagem serve de base para a teoria de Georg Cantor sobre números transfinitos. Segundo Batchelor, no ano de 1874 "Cantor provou a existência de conjuntos infinitos que têm magnitudes diferentes e distintas." ${ }^{1}$ (2002. p.20). Tal trabalho é considerado referência quando nos referimos à Teoria dos Conjuntos. Em termos gerais, podemos afirmar que:

Cantor parte do conceito de conjunto como uma coleção de objetos, que são seus membros, e podendo ser especificado listando seus membros dentre chaves. Se considerarmos, por exemplo, o conjunto dos números reais dentre 1 e 6 podemos escrevê-lo como $\{1,2,3,4$, $5,6\}$, uma alternativa possível é a escrita $\{n: 1 \leq n \leq 6$ e $n$ é um número natural\} (Tavares, 2020. p.2).

O conceito de função remete a "uma receita que começa com algum número e calcula um

${ }^{1}$ Original em inglês: "Cantor had proved the existence of infinite sets that have different and distinct magnitudes." (Batchelor, 2002. p.20). Tradução própria. 
TAVARES, Pedro Henrique Carneiro (2021).

O uso de funções na geração de novas alturas sonoras, a partir de um conjunto de classes de nota.

outro número associado ao primeiro" (Stewart, 2014. p.114), é uma relação entre elementos de dois conjuntos onde para cada valor do primeiro, vamos nomear de $x$, temos um valor no segundo, $f(x)$ ou y. Nos referimos ao conjunto $X$ como domínio e a $F(x)$ ou $Y$ como imagem. Podemos afirmar que o elemento y é o valor que a função $f$ assume no argumento $x$. Desta maneira deixamos evidente que, através de $f$, transformamos $x$ em $y$. De acordo com Halmos "Se $X$ e $Y$ são conjuntos, uma função de (ou em) $\mathrm{X}$ para (ou em) $\mathrm{Y}$ é uma relação $\mathrm{f}$ tal que dom $\mathrm{f}=\mathrm{X}$ e tal que para cada $\mathrm{X}$ em $\mathrm{X}$ há um único elemento y em $Y$ com $(x, y) \in$ f." 2 (2017, p.35). Em outras palavras, para termos uma relação de função entre conjuntos é necessário satisfazer a condição que todos os elementos do conjunto $X$, domínio, estejam relacionados a um único elemento do conjunto $\mathrm{Y}$, imagem.

No referente aos materiais e conceitos extraídos do âmbito musical, são adotadas definições encontradas na Teoria Pós-Tonal, tal qual como exposta por Straus (2013). É importante estabelecer a diferença entre nota e classe de nota, o conceito de nota remete a uma altura sonora específica, como por exemplo o Lá $440 \mathrm{hz}$, enquanto classe de nota trata do conjunto de várias alturas que compartilham a mesma classe, como todas as alturas com nome Lá $(55 \mathrm{hz}, 110 \mathrm{hz}, 220 \mathrm{hz}, 440 \mathrm{hz}$, $880 \mathrm{hz}$, etc). Considerando este conceito temos um total de doze classes sonoras, que podemos nos referir pelos nomes de Dó - Dó\#/Réb - Ré - Ré\#/Mib - Mi - Fá - Fá\#/Solb - Sol - Sol\#/Láb - Lá Lá\#/Sib - Si. Podemos recorrer ao que Straus chama de notação com inteiros, para o intuito deste trabalho, atribuindo o número 0 para a classe de nota Dó, 1 para Dó\#/Réb, 2 para Ré, continuando desta maneira até o número 11 (classe de nota $\mathrm{Si}$ ). Todas as alturas, notas musicais, pertencem a alguma das doze classes de nota ${ }^{3}$, emprega-se, portanto, o módulo 12 (utilizaremos a abreviatura mod12) para o trabalho com as mesmas. Por exemplo, se temos uma nota Lá $440 \mathrm{hz}$, classe de nota 9 , e a elevamos uma oitava para Lá $880 \mathrm{hz}$ temos $9+12=21$, qualquer número que seja menor que zero ou maior que onze é equivalente mod12 a algum inteiro entre zero e onze, 9+12=21 e 21 mod12 $=9$, desta maneira a coerência das classes de nota é mantida.

Um grupo, ou coleção, de classes de nota é referido como Conjunto de classes de nota, tal conceito remete a uma aplicação musical da Teoria dos Conjuntos matemática. No contexto musical temos a Teoria dos Conjuntos de Classes de Nota, que emerge como opção de ferramenta analítica para músicas atonais. Segundo Kostka $(2018$, p.171) notou-se que a música atonal frequentemente alcançava um grau de unidade através do trabalho de um conjunto de classes de nota, um trabalho de referência no assunto se encontra em A estrutura da música atonal, de Allen Forte. 0 conjunto pode ser desenvolvido de diversas maneiras, tais como transposição, inversão, multiplicação. $\mathrm{Na}$ pesquisa realizada, porém, não foram encontrados exemplos de peças, ou trabalhos, aonde o compositor aplicou alguma função para desenvolver o conjunto de classes de nota utilizado. Acreditando ser plenamente possível tal aplicação de funções, foi realizada uma investigação sobre a questão. Neste artigo são apresentados os resultados obtidos.

\footnotetext{
${ }^{2}$ Original em inglês: "If $X$ and $Y$ are sets, a function from (or on) $X$ to (or into) $Y$ is a relation $f$ such that dom $f=X$ and such that for each $x$ in $X$ there is a unique element $y$ in $Y$ with $(x, y) \in f . "$ (Halmos, 2017. p.35). Tradução própria.

${ }^{3}$ A afirmação considera o uso do sistema temperado de afinação, que divide a oitava sonora em doze partes iguais. 
TAVARES, Pedro Henrique Carneiro (2021)

$O$ uso de funções na geração de novas alturas sonoras, a partir de um conjunto de classes de nota.

\section{Material e Métodos}

Os materiais utilizados foram funções, de primeiro e segundo grau, e um conjunto de classes de nota. Basicamente podemos afirmar que o grau da função remete ao maior expoente assumido pela incógnita $x$, temos como modelo padrão para função de primeiro grau a estrutura $f(x)$ $=a x+b$, e para função de segundo grau a estrutura $f(x)=a x^{2}+b x+c$. De acordo com os conceitos previamente expostos, temos por conjunto de classes de nota uma coleção, ou grupo, de classes de nota. Determinamos um conjunto de classes de nota a ser trabalhado, segundo Kostka (2018) e Tavares (2020) o mesmo é nomeado reorganizando suas classes de nota na estrutura mais compacta possível, e as nomeando através da notação com inteiros. Para determinar esta estrutura, mais compacta possível, comparamos também a arrumação mais compacta do conjunto com seu conjunto equivalente por inversão, uma vez que ambos são considerados equivalentes, a opção mais compacta é a escolhida. Resumidamente, podemos realizar o procedimento seguindo as etapas: identificação da coleção de alturas; reorganização das mesmas de maneira a encontrar a estrutura mais compacta, chamada na teoria pós-tonal de ordem normal; inversão do conjunto; colocar a inversão em ordem normal; comparação entre as ordens normais identificando a mais compacta, ou a melhor ordem normal; nomear o conjunto de classe de nota resultante através da notação com inteiros.

Tais procedimentos encontram-se bem exemplificados através de ilustração presente no texto de Pedro e Maria Clara Tavares (2021. p.103). Para manter o foco no objetivo deste trabalho, apresentamos os conjuntos já em sua melhor ordem normal. Na Figura 1 encontra-se apresentado o conjunto utilizado, em sua forma mais compacta, sua melhor ordem normal, e consequente nome do conjunto, $[0 ; 1 ; 2 ; 6]$.

Figura 01: Conjunto $[0 ; 1 ; 2 ; 6]$

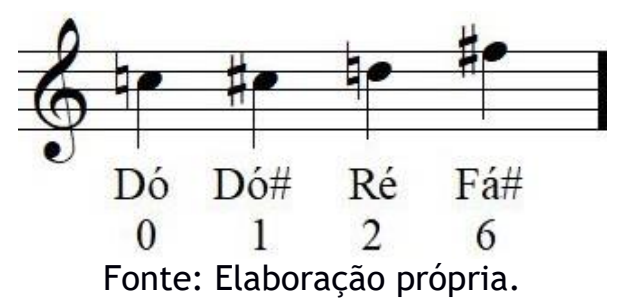

De acordo com procedimentos propostos pela teoria pós-tonal, particularmente Straus (2013), utilizamos a notação com inteiro para as classes de nota. Desta maneira estamos assumindo a seguinte relação entre inteiros e classes de nota: 0 para a classe de nota Dó, 1 para Dó\#/Réb, 2 para Ré, 3 para Ré\#/Mib, 4 para Mi, 5 para Fá, 6 para Fá\#/Solb, 7 para Sol, 8 para Sol\#/Láb, 9 para Lá, 10 para Lá\#/Sib e 11 para Si.

Uma vez estabelecido o nosso conjunto, o método adotado consistiu de, para cada função, atribuir à incógnita $x$ o valor do inteiro correspondente a cada classe de nota do conjunto original. Desta forma, obtendo uma nova coleção de classes, os quais optamos tratar como um novo conjunto. 
TAVARES, Pedro Henrique Carneiro (2021).

$O$ uso de funções na geração de novas alturas sonoras, a partir de um conjunto de classes de nota.

A partir dos resultados obtidos foi construída, com intuito de experimentação e ilustração de possibilidade aplicativa, uma frase musical a partir do conjunto original. Em seguida, a frase foi modificada para se adequar aos novos conjuntos obtidos, desta maneira ilustrando uma possibilidade de variação da mesma que pode ser aplicada no campo de Composição musical.

\section{Material e Métodos}

$\mathrm{Na}$ aplicação da função para gerar novas alturas sonoras, a partir do conjunto de classes de nota, foi atribuída à incógnita $x$ o valor do inteiro referente a cada classe de nota do conjunto. Por tratarmos de classes de nota, estamos adotando o mod12. A partir disto, a função de primeiro grau, $f(x)=a x+b$, assume a forma $f(x)=(a x+b) \operatorname{mod12}$, e a função de segundo grau, $f(x)=a x^{2}+b x+c$, assume $f(x)=\left(a x^{2}+b x+c\right) \bmod 12$

Foram definidos valores para $a, b$ e $c$, com finalidade de aplicar a função no conjunto escolhido, $[0 ; 1 ; 2 ; 6]$. Para $a$ foi atribuído o valor 2 , para $b$ o valor 1 e para $c$ o valor 3 . Aplicando tais valores nossas funções resultantes foram: $f(x)=(2 x+1) \bmod 12$, e $f(x)=\left(2 x^{2}+1 x+3\right) \bmod 12$, que podemos escrever $f(x)=\left(2 x^{2}+x+3\right) \bmod 12$.

A etapa seguinte consiste de aplicar a função em cada classe de nota do conjunto, com finalidade de gerarmos novas classes, ou alturas sonoras. Nosso conjunto é o $[0 ; 1 ; 2 ; 6]$, empregando a função $f(x)=(2 x+1) \bmod 12$ temos:

\section{$[(2.0+1) \bmod 12 ;(2.1+1) \bmod 12 ;(2.2+1) \bmod 12 ;(2.6+1) \bmod 12]$ \\ $[(0+1) \bmod 12 ;(2+1) \bmod 12 ;(4+1) \bmod 12 ;(12+1) \bmod 12]$

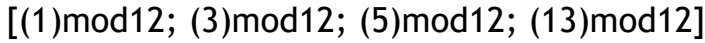

$$
[1 ; 3 ; 5 ; 1]
$$

Descartando a repetição da classe 1 temos: $[1 ; 3 ; 5]$

Um resultado interessante foi a repetição da classe de nota 1, que não precisa ser escrita novamente, logo geramos uma coleção de três classes, [1;3;5] a partir de um conjunto de quatro classes, através da função escolhida. Se há intenção de tratar essa coleção de classes como um conjunto, podemos nomeá-lo. Para tal, é necessário transpô-lo de modo que a primeira classe seja 0 , e encontrar sua melhor ordem normal. No caso de nossa coleção podemos subtrair o intervalo 1 para cada classe de nota, [(1-1);(3-1);(5-1)], de maneira que obtemos agora a mesma representada a partir da classe 0: [0;2;4], essa também corresponde à sua melhor ordem normal e, portanto, é o nome do conjunto. 0 mesmo procedimento foi efetuado a partir da nossa função de segundo grau, $f(x)=\left(2 x^{2}+1 x+3\right) \bmod 12:$

$$
\begin{gathered}
{\left[\left(2.0^{2}+1.0+3\right) \bmod 12 ;\left(2.1^{2}+1.1+3\right) \bmod 12 ;\left(2.2^{2}+1.2+3\right) \bmod 12 ;\left(2.6^{2}+1.6+3\right) \bmod 12\right]} \\
{[(0+0+3) \bmod 12 ;(2+1+3) \bmod 12 ;(8+2+3) \bmod 12 ;(72+6+3) \bmod 12]} \\
{[(3) \bmod 12 ;(6) \bmod 12 ;(13) \bmod 12 ;(81) \bmod 12]} \\
{[3 ; 6 ; 1 ; 9]}
\end{gathered}
$$


TAVARES, Pedro Henrique Carneiro (2021)

$O$ uso de funções na geração de novas alturas sonoras, a partir de um conjunto de classes de nota.

Reorganizando: $[1 ; 3 ; 6 ; 9]$

Aplicando a função $f(x)=\left(2 x^{2}+1 x+3\right) \bmod 12$ obtivemos as classes de nota [1;3;6;9], a partir do conjunto $[0 ; 1 ; 2 ; 6]$. Para tratarmos essa coleção como conjunto vamos transpô-la para partirmos da classe 0 , igualmente subtraindo o intervalo 1 , [(1-1);(3-1);(6-1);(9-1)]. Obtemos assim o conjunto $[0 ; 2 ; 5 ; 8]$. já na melhor ordem normal. Através da Figura 02 estão ilustrados o conjunto original e os novos conjuntos gerados.

Figura 02: Conjunto original e novos conjuntos gerados.

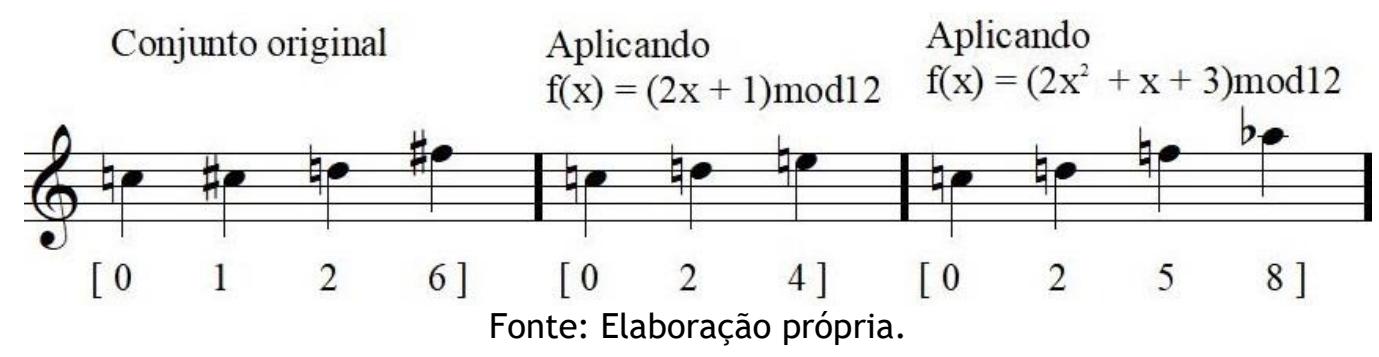

É exposta a seguir uma frase musical construída a partir do conjunto original, $[0 ; 1 ; 2 ; 6]$, seguida por variações da mesma através dos novos conjuntos, [0;2;4] e [0;2;5;8]. O objetivo é ilustrar uma possibilidade de variação a partir da geração de novas classes empregando funções em um conjunto original, técnica de variação tal que pode ser aplicada no âmbito de composição musical. A Figura 03 ilustra a frase desenvolvida e as suas variações, nomeamos de frase $a$, $a$ ' e $a$ ". A adoção de apóstrofos, ao invés de utilizar outra letra, como $b$ e $c$, ocorre por se tratar de variações da frase $a$. Foi procurado manter um bom nível de semelhança entre a frase $a$ e suas variações, particularmente em relação ao contorno melódico e a rítmica, focando a atenção em destacar as alturas sonoras geradas a partir do procedimento proposto. As frases $a$ e $a$ " partem de conjuntos com quatro membros, enquanto $a$ ' utiliza um conjunto com três membros, musicalmente isso foi retratado através do uso de compasso quaternário para $a$ e $a$ ", enquanto $a$ ' é escrito em compasso ternário, Os conjuntos foram trabalhados de forma a estarem interligados, ao menos um membro de cada apresentação do conjunto pertencendo também a outra apresentação do mesmo, assim interligando o trabalho das alturas na frase musical. Os conjuntos encontram-se destacados, para fácil identificação, na Figura 03.

Figura 03: Frases musicais. 
TAVARES, Pedro Henrique Carneiro (2021).

O uso de funções na geração de novas alturas sonoras, a partir de um conjunto de classes de nota.

Frase a - Conjunto $[0 ; 1 ; 2 ; 6]$

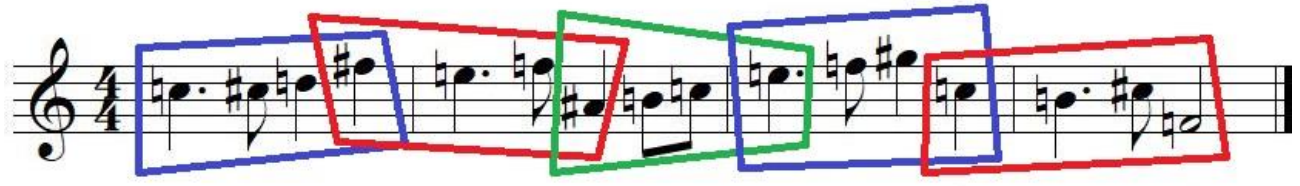

Frase a' - Conjunto $[0 ; 2 ; 4]$

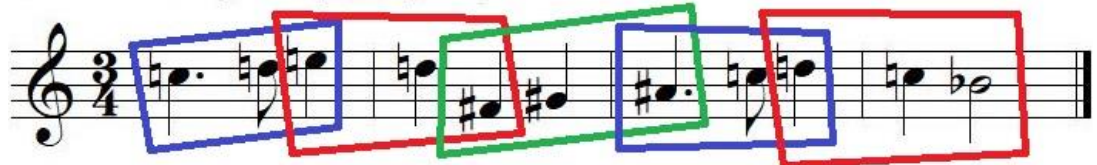

Frase a" - Conjunto $[0 ; 2 ; 5 ; 8]$

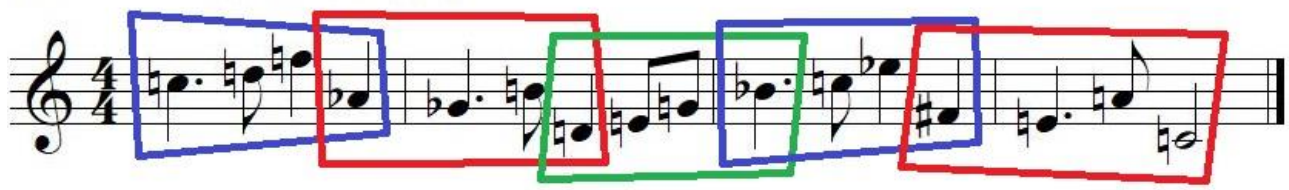

Fonte: Elaboração própria.

Nota-se que as frases $a^{\prime}$ e $a$ " guardam semelhanças rítmicas e de contorno melódico com a frase $a$, que foram mantidas de maneira intencional, focando o trabalho apenas nas alturas sonoras. Tais frases, $a^{\prime}$ e $a$ ", são opções viáveis de variações a serem utilizadas na construção de uma peça musical. É igualmente possível não resguardar tais semelhanças, tornando as variações ainda mais contrastantes em relação a frase original.

Demonstramos a técnica através de uma melodia desacompanhada, porém a mesma lógica pode ser aplicada em contextos polifônicos de diversas naturezas, tais como contraponto imitativo, melodia acompanhada, etc.

\section{Conclusões}

A proposta partiu da observação quanto às principais abordagens referentes ao trabalho com um conjunto de classes de nota na construção de peças musicais. As técnicas mais frequentes se configuram no uso de transposição, inversão, multiplicação, permutação, dentre outras. Outras opções são encontradas, porém em menor quantidade de vezes seu uso é efetivamente realizado por compositores. Na pesquisa realizada não foram encontrados nenhum caso aonde alguma função foi empregada no trabalho com as classes de nota, essa constatação constituiu a motivação em iniciar o trabalho.

A partir dos resultados alcançados pode-se concluir que a aplicação de funções, na geração de novas classes a partir de um conjunto de classes de nota original, é não apenas possível, como gera opções bastante viáveis de ferramentas para criação musical. Através do trabalho de variação, a partir de uma frase musical construída, foi demonstrada uma técnica viável de aplicação, sabendo que outras são igualmente possíveis de serem exploradas por compositores. Se espera que o presente artigo constitua um veículo através do qual os resultados expostos ofereçam novas ferramentas a serem utilizadas por compositores, que desejem novas formas de desenvolverem ideias musicais a partir da teoria dos conjuntos de classes de nota. 
TAVARES, Pedro Henrique Carneiro (2021)

O uso de funções na geração de novas alturas sonoras, a partir de um conjunto de classes de nota.

\section{Referências}

BATCHELOR, John H. The History and Evolution of the Concept of Infinity. 2002. Disponível em: https://www.academia.edu/15202414/The_History_and_Evolution_of_the_Concept_of_Infinity?emai L_work_card=title. Acesso em: 17/09/2019.

HALMOS, Paul R. Naive set theory. New York: Dover Publications, 2017.

KOSTKA, Stefan M; SANTA, Matthew. Materials and techniques of post-tonal music. 5.ed. New York: Routledge, 2018.

MENEZES, Flo. Apoteose de Schoenberg. 2.ed. Ateliê Editorial, 2002.

STEWART, lan. Em busca do infinito: Uma história da matemática dos primeiros números à teoria do caos. Tradução de George Schlesinger. Rio de Janeiro: Zahar, 2014.

STRAUS, Joseph Nathan. Introdução à teoria pós-tonal. 3.ed. Tradução de Ricardo Bordini. UNESPEDUFBA, Salvador-São Paulo, 2013.

TAVARES, Pedro. Breve introdução a Teoria dos Conjuntos de Classes de Nota. 2020.

Disponível em:

https://www.academia.edu/41907195/Pedro_Tavares_Breve_introdu\%C3\%A7\%C3\%A3o_a_Teoria_dos_ Conjuntos_de_Classes_de_Nota_2020_

Acesso em: 18/08/2020.

TAVARES, Pedro; TAVARES, Maria Clara de Sousa. Composição musical a partir de Modelagem Sistêmica e Teoria dos Conjuntos. Ictus Music Journal, v. 15, n.1, p. 95-108. 2021.

Disponível em: Composicão musical a partir de Modelagem Sistêmica e Teoria dos Conjuntos | ICTUS - Periódico do PPGMUS-UFBA | ICTUS Music Journal

Acesso em: 11/10/2021. 